\title{
Percutaneous coronary revascularization in diabetics
}

\author{
Francesco Pollice ${ }^{1}$, Paolo Pollice ${ }^{1}$, Lyan Jacob ${ }^{1}$
}

${ }^{1)}$ Department of Cardiology And Interventional Radiology. University Hospital Basel, Switzerland

\section{Summary}

Objective: Diabetics with coronary artery disease face a high risk of adverse events following coronary revascularization. However, recurrence rates of after the first revascularization have never been appraised. The aim of this study was to evaluate recurrent events in diabetics undergoing percutaneous coronary intervention (PCI) in the current era.

Material and Method: Authors collected baseline and outcome data of consecutive type-2 diabetics treated with PCI (January 2005-December 2008). End-points of interest were the long-term rates of major adverse cardiac events (MACE: cardiac death, myocardial infarction [MI], percutaneous target vessel revascularization [TVR-PCI], or coronary artery bypass grafting [CABG]), non-TVR PCI, and stent thrombosis.

Results: A total of 429 diabetics were included, 191 (44\%) insulin-dependent, with drug-eluting stents implanted in 232 (54\%). After a median of 38 months, events were as follows: MACE in 167 (38.9\%) subjects, cardiac death in 38 (8.8\%), MI in 42(9.8\%), TVR PCI in 130 (30.3\%), CABG in 11 (6.2\%), non-TVR PCI in 52 (12.1\%), and definite stent thrombosis (2.1\%). Among the 129 patients undergoing TVR PCI as first event, as many as 28 (21.7\%) underwent a second TVR PCI, 7 (5.4\%) underwent a third TVR PCI, and a further $2(1.5 \%)$ underwent a fourth TVR PCI, whereas CABG was performed in 2 (1.5\%)and non-TVR PCI in 4 (3.1\%).

Conclusion: This work, originally reporting on risk of recurrent repeat revascularization events among diabetics treated with PCI, showed that adverse events occur frequently in these patients, but can be managed in most cases safely and successfully by means of repeat PCI only.

Keywords: Stroke, coronary artery disease, diabetics.

\section{Introduction}

Many complications develop in diabetics: microvascular complications, including retinopathy, nephropathy, and neuropathy, and macrovascular complications, and including cardiac, cerebrovascular, and peripheral vascular complications ${ }^{[1]}$ Cardiovascular disease is the principal cause of death and disability in people with diabetes as it recognizes a unique pathophysiology. ${ }^{[2]}$ Several features of atherosclerosis make diabetic patients 2 to 4 times more likely to develop coronary artery disease than non-diabetic patients and to manifest this condition earlier in life. moreover, many observational and experimental data showed a worse prognosis for diabetics experiencing a myocardial infarction 
(MI) or undergoing coronary revascularization. ${ }^{[3]}$ The presence of several comorbidities, multivessel and diffuse coronary disease with small vessel diameter, long lesions and plaques prone to rupture, together with accelerated atherosclerosis progression, compromises periprocedural and long-term outocomes of both coronary artery bypass grafting or percutaneous coronary intervention (PCI) ${ }^{[4]}$ Indeed, owing to its unique pathophysiologic milieu and unfavorable anatomical pattern, diabetes makes either revascularization approach seemingly suboptimal. Diabetic patients thus clearly need, in addition to optimal medical therapy, various therapeutic strategies for myocardial ischemia tailored to the different stages of disease.

In such a setting, many studies have explored the long-term risk of single or first ever adverse events after coronary revascularization in diabetics,${ }^{[5]}$ but none sistematically appraised to date the risk of date the risk of recurrent and repeat adverse events, e.g., PCI followed in the same patient by bypass grafting and then another PCI. We thus aimed to retrospectively assess intermediate and long-term recurrences of major adverse cardiac events and repeat target vessel revascularizations in a cohort of diabetic subjects undergoing PCI.

\section{Materials and methods}

Specifically, a series of 429 consecutive patients were enrolled according to the following criteria: 1) previous or new diagnosis of type 2 diabetes mellitus according to contemporary American Diabetes Association criteria, ${ }^{[6]}$ with patients classified as "non-insulin requiring", including patients treated with diet and/or oral hypoglycemic drugs but no insulin, and "insulin requiring", including patients treated with insulin regardless of other therapies; and 2 treatment with PCI between July 1, 2002, and December 31, 2005 at our Center, with implantation of bare-metal stents and/or drug eluting stents. Age, acute ST-elevation MI, combined treatment with the implantation of both bare-metal and drug eluting stents in the index procedure were not exclusion criteria.

All patients completed a written informed consent form, and institutional Ethic Committee approval was waived given the observational design of the study. All procedures were performed according to standard guidelines for PCI, ${ }^{[7]}$ but interventional strategy and choice of device were decided by the attending physician Patients who were not chronically receiving aspirin, were pretreated with a $500 \mathrm{mg}$ oral loading dose at least three hours before the procedure, or a $300 \mathrm{mg}$ intravenous loading dose directly before the procedure. Premedication included also a loading dose of $300 \mathrm{mg}$ of clopidogrel the day before a planned procedure or $600 \mathrm{mg}$ at least two hours before a procedure for nonST- elevation acute coronary syndrome. All patients received intra-arterial unfractioned heparin to maintain an activated clotting time longer than 250 seconds.

The use of glycoprotein IIb/IIIa inhibitors was left at the operator's preference. Angiographic success was defined as residual diameter stenosis $<30 \%$ by visual estimate in the presence of a thrombolysis in MI grade 3 flow. Revascularization was considered functionally complete in case of effective treatment of all epicardial vessel with diameter $>2.25 \mathrm{~mm}$, supplying viable areas of left ventricular myocardium, and affected by a diameter stenosis $75 \%$ by visual estimate. After the procedure all patients were prescribed lifelong aspirin (70-325 mg/d). In addition, one month of clopidogrel $(75 \mathrm{mg} / \mathrm{d})$ or ticlopidine $(250 \mathrm{mg}$ twice a day) was recommended for patients treated with bare-metal stents, at least three-six months following drug-eluting stent implantation, and, in any case, at least 9-12 months following a non-ST-elevation acute coronary syndrome.

The primary outcome measure of this study was the cumulative incidence in hospital and at follow-up of major adverse cardiac events (MACE), defined as the composite of 1) cardiac death (all death were considered cardiac unless an unequivocal non-cardiac cause could be established); 2) MI; ${ }^{[8]} 3$ ) target vessel revascularization (TVR, any repeat percutaneous intervention or surgical bypass of any segment of the target vessel). The target vessel was defined as the entire major coronary vessel proximal and distal to the target lesion, which includes upstream and downstream branches and the target lesion itself. Any percutaneous procedure planned to complete the index revascularization was considered part of the index procedure, not a repeat revascularization.

Predefined secondary outcome measures were: 1 ) non cardiac death (non-cardiovascular death or vascular non- 
cardiac death); 2) MI; 3) percutaneous TVR; 4) percutaneous non -TVR ( i.e., a repeat percutaneous intervention driven by any lesion located in a epicardial vessel ferent from the target vessel); 4) surgical revascularization by means of coronary artery bypass grafting; 5) stroke; 6) stent thrombosis (divided into definite, probable and possible according to the Academic Research Consortium recommendations) ${ }^{[9]}$ For each patient, baseline demographic data, cardiovascular risk profile, treatment for risk factor control, other medical history data, as well as procedural and angiographic follow-up details were obtained from the institutional database, visits at the outpatient clinic or telephone interviews. All previous mentioned data were collected using individualized case report forms and entered into a dedicated database.

\section{Statistical analysis}

Continuous variable are presented as mean standard deviation or median and interquartile range. Student's test and/or analysis of variance were performed to determine differences between mean values. Categorical variables are reported as absolute values and percentage with $95 \%$ confidence intervals, with comparisons made with the chi-square test or Fisher exact test where appropriate. Two-tailed probability values $<0.05$ were considered statistically significant. Data were analysed with CIA, EpiInfo ad SPSS softwares.

TABLE I.-Baseline clinical characteristics of patients according to treatment group.

\begin{tabular}{|c|c|c|c|c|c|}
\hline Variable & $\begin{array}{c}\text { DES } \\
(\mathrm{N} .=133)\end{array}$ & $\begin{array}{c}\text { Both } \\
(\mathrm{N} .=99)\end{array}$ & $\begin{array}{c}\text { BMS } \\
(\mathrm{N},=197)\end{array}$ & $\begin{array}{l}\text { Overall } \\
(\mathrm{N},=429)\end{array}$ & P \\
\hline Age $($ mean $\pm S D), y$ & $64.8 \pm 10.6$ & $65.9 \pm 10.0$ & $69.7 \pm 9.5$ & $67.3 \pm 10.2$ & 0.0001 \\
\hline Male gender N. (\%) & $86(65)$ & $74(75)$ & $138(70)$ & $298(69)$ & 0.2489 \\
\hline Duration of diabetes (mean $+D S), y$ & $14.9 \pm 10.0$ & $16.8 \pm 9.8$ & $14.2 \pm 10.2$ & $16.7 \pm 10.9$ & 0.3395 \\
\hline Ejection fraction (mean $\pm D S), \%$ & $54.3 \pm 10.7$ & $49.9 \pm 11.6$ & $50.6 \pm 12.0$ & $52.0 \pm 11.6$ & 0.0070 \\
\hline Hypertension N. (\%) & $117(88)$ & $91(92)$ & $171(87)$ & $379(88)$ & 0.4269 \\
\hline Dyslipidemia N. (\%) & $108(81)$ & $76(77)$ & $139(71)$ & $323(75)$ & 0.0826 \\
\hline Family history of coronary disease N. (\%) & $26(20)$ & $12(12)$ & $37(19)$ & $75(17)$ & 0.2729 \\
\hline Current smoker N. (\%) & $13(10)$ & $16(16)$ & $27(14)$ & $56(13)$ & 0.3368 \\
\hline Former smoker N. (\%) & $54(40)$ & $37(37)$ & $80(41)$ & $171(40)$ & 0.8470 \\
\hline Body mass index (mean $\pm \mathrm{SD}) \mathrm{kg} / \mathrm{mC}$ & $27.8 \pm 4.8$ & $27.5 \pm 3.8$ & $28.1 \pm 4.5$ & $27.9 \pm 4.4$ & 0.7200 \\
\hline \multicolumn{6}{|l|}{ Renail failure N. (\%) } \\
\hline Serum creatinine? $2 \mathrm{mg} / \mathrm{dL}$ & $10(8)$ & $10(10)$ & $23(12)$ & $43(10)$ & 0.4672 \\
\hline Dialysis & $3(2)$ & $5(5)$ & $9(5)$ & $17(4)$ & 0.4685 \\
\hline Peripheral vasculopathy N. (\%) & $19(14)$ & $28(28)$ & $60(30)$ & $107(25)$ & 0.0026 \\
\hline Chronic obstructive pulmonary disease N. (\%) & $4(3)$ & $6(6)$ & $14(7)$ & $24(5)$ & 0.2754 \\
\hline Severe valvulopaty N. (\%) & $6(4)$ & $4(4)$ & $20(10)$ & $30(7)$ & 0.0605 \\
\hline Previous myocardial infarction N. (\%) & $47(35)$ & $39(39)$ & $87(44)$ & $173(40)$ & 0.2704 \\
\hline Previous coronary angioplasty N. (\%) & $43(32)$ & $24(24)$ & $43(22)$ & $110(26)$ & 0.0941 \\
\hline Previous bypass surgery N. (\%) & $22(16)$ & $22(22)$ & 35 (18) & $79(18)$ & 0.5165 \\
\hline Previous stroke N. (\%) & $4(3)$ & $3(3)$ & $9(4)$ & $16(4)$ & 0.6997 \\
\hline \multicolumn{6}{|l|}{ Admission diagnosis N. (\%) } \\
\hline Stable angina or silent ischemia & $35(26)$ & $35(35)$ & $56(28)$ & $126(29)$ & 0.3025 \\
\hline Unstable angina & $64(48)$ & $28(28)$ & $62(31)$ & $154(36)$ & 0.0016 \\
\hline Non-ST-elevation myocardial infarction & $21(16)$ & $20(20)$ & $46(23)$ & $87(20)$ & 0.2456 \\
\hline ST-elevation myocardial infarction & $13(10)$ & $16(16)$ & $33(17)$ & $62(14)$ & 0.1799 \\
\hline \multicolumn{6}{|l|}{ Medical therapy N. (\%) } \\
\hline Oral antidiabetic agents & $62(47)$ & $43(43)$ & $81(41)$ & $186(43)$ & 0.6131 \\
\hline Insulin & $57(43)$ & $46(46)$ & $92(47)$ & $195(46)$ & 0.7687 \\
\hline $\begin{array}{l}\text { Angiotensin-converting enzyme inhibitors or angiotensin- } \\
\text { receptor blockers }\end{array}$ & $99(74)$ & $67(68)$ & $124(63)$ & $290(67)$ & 0.0912 \\
\hline Calcium-channel blockers & $33(25)$ & $25(25)$ & $51(26)$ & $109(25)$ & 0.9752 \\
\hline blocker & $114(86)$ & $73(74)$ & $138(70)$ & $325(76)$ & 0.0043 \\
\hline Nitrates & $34(25)$ & $31(31)$ & $64(32)$ & $129(30)$ & 0.3858 \\
\hline Statins & $87(65)$ & $63(63)$ & $109(55)$ & $259(60)$ & 0.1389 \\
\hline Antiplatelet agents & $129(97)$ & $97(98)$ & $179(91)$ & $405(94)$ & 0.0125 \\
\hline $\begin{array}{l}\text { Dual antiplatelet therapy duration after index procedure } \\
\text { (mean } \pm \text { SD), months }\end{array}$ & $5.0 \pm 2.7$ & $4.9 \pm 2.6$ & $1.0 \pm 2.6$ & $3.2 \pm 2.6$ & $<0.0001$ \\
\hline
\end{tabular}

Defined as combined treatment with bare-metal stents (BMS) and drug eluting stents (DES). 


\section{Results}

A total of 429 subjects met the study inclusion criteria. Baseline characteristics according to sent type are presented in Table I. Overall, age was 67.310 .2 years, and $69 \%$ of the patients were male. The clinical characteristics of the present cohort of patients than for diabetics in previously reported studies. Indeed, $88 \%$ (379 patients) had hypertension, 75\% (323) had dyslipidemia, and their body mass index was $27.94 .4 \mathrm{~kg} / \mathrm{m}^{2}$. Insulin-requiring diabetes was observed in $46 \%$ (195) of subjects and often the index procedure was not their first original revascularization procedure, as $40 \%$ (173) had experienced, as 40\% (173) had experienced a previous MI, 26\% (110) a previous coronary angioplasty and $18 \%$ (79) a prior bypass surgery. Diagnosis at admission was stable angina or silent ischemia for $29 \%$ (126) of patients. Compared with subject who received drugeluting stents, those who received bare-metal stents had a more severe clinical profile: they were older $(\mathrm{P}<0.01)$, ha poorer left ventricular ejection fraction $(\mathrm{P}<0.01)$ and more diffuse atherosclerosis (ie concomitant peripheral artery disease, $\mathrm{P}<0.01$ ). The duration of dual antiplatelet therapy was 5.0 2.7 month after drug-eluting stent implantation, 1.0 2.7 month after bare-metal stenting, and 4.9 2.6 months after combined treatment with both drug-eluting and bare-metal stents. In $6 \%$ (24) of cases dual antiplatelet therapy was not administered at dis- charge because of drug intolerance.

As show in Table II, multivessel disease was present in $73.6 \%$ (316) of subjects, 31.7 lesions were treated per patient, and 31.8 stents were implanted per patient. Concerning stent type, 197 (46\%) received only baremetal stents, $133(31 \%)$ received only drug-eluting stents (sirolimus or paclitaxel-eluating stents), and 99 (23\%) received both bare-metal and drug-eluating stents. There were significant differences between these groups in terms of angiographic characteristics, with a more complex procedural profile for patients treated with both stent types, and an excess of drug-eluting stent implantation at bifurcation lesions and unprotected left main (all $\mathrm{P}<0.05$ ). Follow-up duration was thus 41.0 12.2 months (median 38.5 months, interquartile renge).

The three-year cumulative incidence of MACE was $38.9 \%$ (167) with a significantly better outcome in the drug-eluting stent only group. The overall rate of MI was $9.8 \%$ (42), with only $1(0.2 \%)$ patient facing a second MI. Revascularization procedures occurred as follows: bypass grafting in $2.6 \%$ (11), first percutaneous TVR in $6.7 \%$ (29), third percutaneous interventions on epicardial vessels not previously treated: $1.1 \%$ (52) of patients underwent a first percutaneous non TVR, $1.6 \%(7)$ a second percutaneous non TVR, $0.5 \%$ (2) a third percutaneous non- TVR. Comparisons between groups showed that patients treated with bare-metal

TABLE II.-Angiograpbic and procedural characteristics according to treatment group.

\begin{tabular}{|c|c|c|c|c|c|}
\hline Variable & $\begin{array}{c}\text { DES } \\
(\mathrm{N} .=133)\end{array}$ & $\begin{array}{c}\text { Both } \\
(\mathrm{N} .=99)\end{array}$ & $\begin{array}{c}\text { BMS } \\
(\mathrm{N},=197)\end{array}$ & $\begin{array}{c}\text { Overall } \\
(\mathrm{N} .=429)\end{array}$ & P \\
\hline Multivessel disease N. (\%) & $92(69.2)$ & $90(90.9)$ & $134(68.0)$ & $316(73.6)$ & $<0.0001$ \\
\hline N. of ciseased vessels/patient (mean \pm SD) & $2.0 \pm 0.8$ & $2.4 \pm 0.6$ & $2.0 \pm 0.8$ & $2.1 \pm 0.8$ & $<0.0001$ \\
\hline N. of treated vessels/patient (mean $\pm S D)$ & $1.6 \pm 0.9$ & $2.1 \pm 0.6$ & $1.6 \pm 0.7$ & $2.0 \pm 0.7$ & 0.0001 \\
\hline N. of treated lesions/patient (mean \pm SD) & $2.6 \pm 1.6$ & $4.1 \pm 1.8$ & $2.5 \pm 1.4$ & $3.0 \pm 1.7$ & $<0.0001$ \\
\hline \multicolumn{6}{|l|}{ Vessel treated N. (\%) } \\
\hline Left anterior descending & $84(63.1)$ & $83(83.8)$ & $117(59.4)$ & $284(66.2)$ & 0.0001 \\
\hline Left circumflex & $64(48.1)$ & $67(67.7)$ & $104(52.8)$ & $235(54.8)$ & 0.0093 \\
\hline Right coronary artery & $53(39.8)$ & $47(47.5)$ & $86(43.6)$ & $186(43.3)$ & 0.5074 \\
\hline Venous graft & $3(2.2)$ & $4(4.0)$ & $15(7.6)$ & $22(5.1)$ & 0.0821 \\
\hline Unprotected left main N. (\%) & $14(10.5)$ & $17(17.2)$ & $5(2.5)$ & $36(8.4)$ & $<0.0001$ \\
\hline Chronic total occlusion N. (\%) & $24(18.0)$ & $25(25.5)$ & $36(18.3)$ & $85(19.8)$ & 0.3013 \\
\hline In-stent restenosis N. (\%) & $18(13.5)$ & $7(7.1)$ & $15(7.6)$ & $40(9.3)$ & 0.1310 \\
\hline Bifurcation lesions N. (\%) & $32(24.1)$ & $27(27.3)$ & $28(14.2)$ & $87(20.3)$ & 0.0132 \\
\hline Stent implanted/patient (mean $\pm S D$ ) & $2.4 \pm 1.8$ & $4.0 \pm 1.7$ & $2.4 \pm 1.6$ & $3.0 \pm 1.8$ & 0.0001 \\
\hline
\end{tabular}

Defined as combined treatment with bare-metal stents (BMS) and drug eluting stents (DES). 
TABLE III.-In-bospital outcomes according to treatment group.

\begin{tabular}{|c|c|c|c|c|c|}
\hline Variable & $\underset{\substack{\text { DES } \\
(\mathrm{N}=133)}}{\text { DES }}$ & $\begin{array}{c}\text { Both } \\
(\text { N. }-99)\end{array}$ & $\begin{array}{c}\text { BMS } \\
(\mathrm{N} .=197)\end{array}$ & $\begin{array}{l}\text { Overall } \\
(\mathrm{N},-429)\end{array}$ & $\mathrm{P}$ \\
\hline Major adverse cardiac events† N. (\%) & $3(2.2)$ & $13(13.1)$ & $25(12.7)$ & $41(9.5)$ & 0.0026 \\
\hline Death N. $(\%)$ & $0(0)$ & $0(0)$ & $5(2.5)$ & $5(1.2)$ & 0.0508 \\
\hline Myocardial infarction N. (\%) & $3(2.2)$ & $13(13.1)$ & $20(10.1)$ & $36(8.4)$ & 0.0060 \\
\hline Coronary bypass grafting N. (\%) & $0(0)$ & $1(1.0)$ & $0(0)$ & $1(0.2)$ & 0.1881 \\
\hline Re-PCI N. $(\%)$ & $0(0)$ & $1(1.0)$ & $3(1.5)$ & $4(0.9)$ & 0.3675 \\
\hline Stroke N. (\%) & $0(0)$ & $0(0)$ & $2(1.0)$ & $2(0.5)$ & 0.3063 \\
\hline
\end{tabular}

"Defined as combined treatment with bare-metal stents (BMS) and drug eluting stents (DES); †Defined as cardiac death, myocardial infarction, target vessel revascularization percutaneous coronary intervention (PCI) or coronary bypass grafting.

stents had a significantly higher cardiac $(\mathrm{P}=0.03)$ and non cardiovascular mortality $(\mathrm{P}=0.01)$, data to be read together with their older age at the time of index procedure $(\mathrm{P}<0.01)$ and longer follow-up duration $(\mathrm{P}<0.01)$.

Further differences were assessed in the number of angiographic follow-up procedures $(\mathrm{P}<0.01)$, percutaneous TVR $(\mathrm{P}<0.01)$ and percutaneous non TVR $(\mathrm{P}<0.01)$ performed in the drug-eluating stent group versus the bare-metal stent counterpart.

We adjudicated 9 (2.1\%) definite stent thromboses: 4 $(0.9 \%)$ occurred within 30 days from index procedures (early), and $5(1.2 \%)$ occurred following this period (late or very ate). Of note, no difference in incidence of definite, probable and possible stent thrombosis was detected among stent type groups $(\mathrm{P}=0.09, \mathrm{P}=0.88$ and $\mathrm{P}=0.55$ respectively). Finally, subgroup analysis showed a higher frequency of all causae death $(22.6 \%$ vs $9.8 \%, \mathrm{P}<0.01)$ and $\mathrm{MI}(13.8 \%$ vs $6.4 \%, \mathrm{P}<0.01)$ for insulin requiring diabetics compared to non insulin requiring patients. (Figures 1-5) allow to follow the assignment of study population to all possible outcomes, particularly to follow the real sequence of revascularization events.

Specifically, following the index procedure the first event was a percutaneous TVR in 30.1\% (129) (95\% CI: 25.9-34.6), bypass grafting in $2.1 \%$ (9) (95\%CI:1.1-3-9), and percutaneous non TVR in $65 \%$ (28) (95\%CI:4.6-9.3), with following-up showing $42.2 \%$ (181) (95\% CI:37.6-46.9) of patients alive without repeat revascularizations, $11.2 \%$ (48) (95\% CI: 8.514.5) dead without repeat revascularizations, $7.9 \%$ (34) (95\% CI:5.7-10.9) untraceable (figure 1). Among 129 patients undergoing percutaneous TVR as first event, $21.7 \%$ (28) (95\% CI: 15.5-29.6) underwent a further percutaneous TVR, 0.8\% (1) (95\% CI:0.1-4-3) experienced bypass as second final event, $1.5 \%$ (2) $(95 \% \mathrm{CI}$ : 0.4-5.5) underwent a percutaneous non-TVR followed in one case $(0.8 \%)(95 \% \mathrm{CI}: 0.1-4.3)$ by a third identical event (Figure 2). Subjects free from further revascularizations were $65.1 \%$ (84) (95\% CI: 56-72.8) living were $65.1 \%$ (84) (95\% CI: 6.6-17.4) deceased.

In the group undergoing percutaneous TVR as second event, a third percutaneous TVR was performed in $25.0 \%$ (7) (95\% CI: 12.7-43.4) of patients, $7.1 \%$ (2) (95\% CI: 2.0-22.6) of them underwent a fourth percutaneous TVR, and 17.8\% (5) (95\% CI: 7.9-35.6) were alive without further interventions (Figure 3). In only one case $(3.6 \%)$ (95\% CI: 0.6-17.7) the third event was surgical revascularization, whereas another patient (3.6\%) (95\% CI:0.6-17.7) underwent percutaneous non-TVR with a subsequent fourth analogous event. Surviving patients without events were $60.7 \%$ (17) (95\% CI: 42.4-76.4), and deceased without revascularization $7.1 \%$ (2) (95\% CI: 2.0-22.6).

During the follow-up 28 subjects underwent only percutaneous revascularizations of epicardial vessels different from the target vessel: $10.7 \%$ (3) (95\% CI: 3.727.2) of them needed a second percutaneous non-TVR, and $3.6 \%$ (1) (95\% CI: 0.6-17.7) a third percutaneous non-TVR (Figure 4). Of the remaining patients $82.1 \%$ (23) (95\% CI: 64.4-92.1) were alive without revascularization, $7.1 \%$ (2) (95\% CI: 2.0-22.6) died without events.

Surgical revascularization occurred as a first event following index revascularization in $2.1 \%$ (9) of cases 
(Figure 5). Among them one patient (11.1\%) (95\% CI: 2.0-43.5) had a second and a third event, both percutaneous TVR. In another one (11.1\%) (95\% CI: 2.0-43.5) percutaneous non-TVR was performed as second event. No second event. No second bypass grafting took place and one patient (11.1\%) (95\% CI: 2.0-43.5) deceased without other revascularizations, whereas $6(66.6 \%)(95 \%$ CI: 35.4-87.9) were alive without further events.

\section{Discussion}

The main findings of this study, appraising the prob- lem of recurrent events in diabetics undergoing percutaneous revascularization, are as follows: 1) repeat target vessel and non-target vessel revascularization occur frequently in this patient population, but can be managed in a safe and successful fashion by means of repeat PCI in most cases; 2) the sequence of revascularization events may be very complex in a minority of subjects, thus the treatment of any new adverse event should be individualized and clinically driven, but also based on coronary anatomy and addressed to the particular stage of diabetic disease; 3) careful clinical fol-

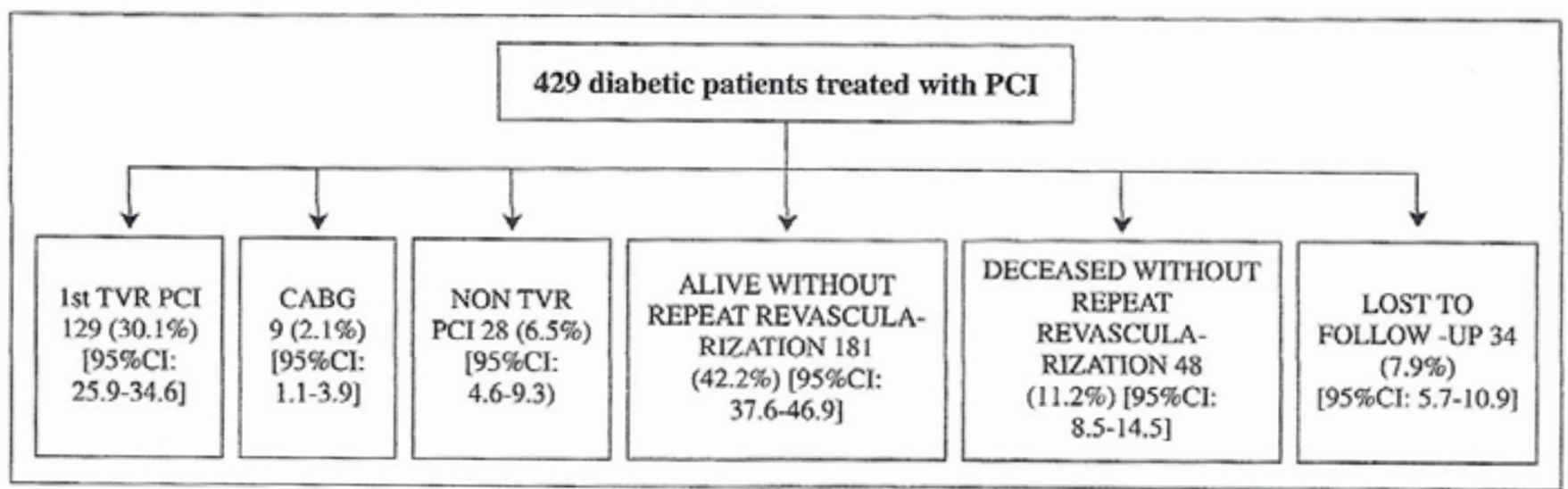

Figure 1.-Occurrence of a first cardiovascular adverse events, reported as N., \%, and 95\% confidence interval. CABG: coronary artery bypass grafting; NON TVR PCI: non target vessel revascularization percutaneous coronary intervention; TVR PCI: target vessel revascularization percutaneous coronary intervention.

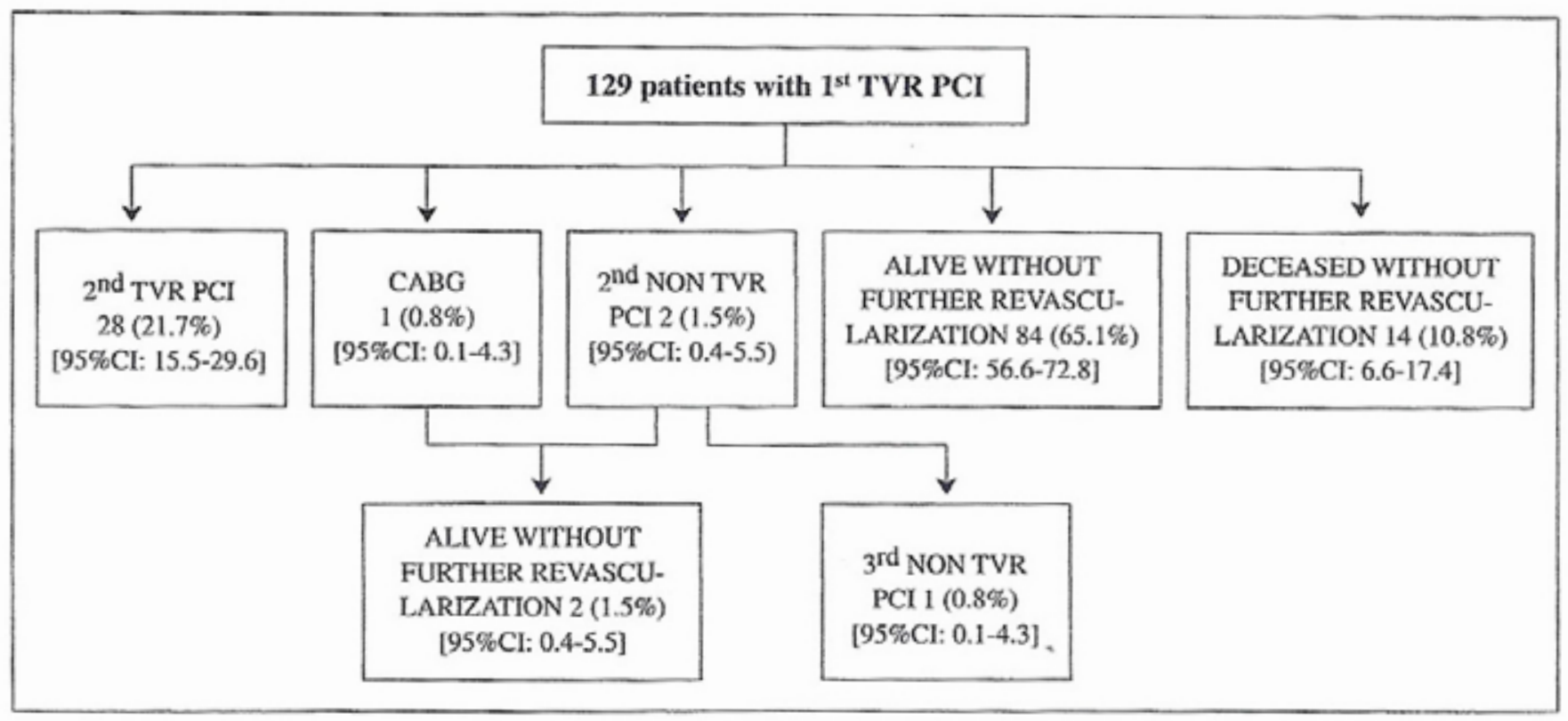

Figure 2.-Need of new revascularization procedures following a first repeat percutaneous coronary intervention, reported as N., \%, and $95 \%$ confidence interval. CABG: coronary artery bypass grafting; NON TVR PCI: non target vessel revascularization percutaneous coronary intervention; TVR PCI: target vessel revascularization percutaneous coronary intervention. 


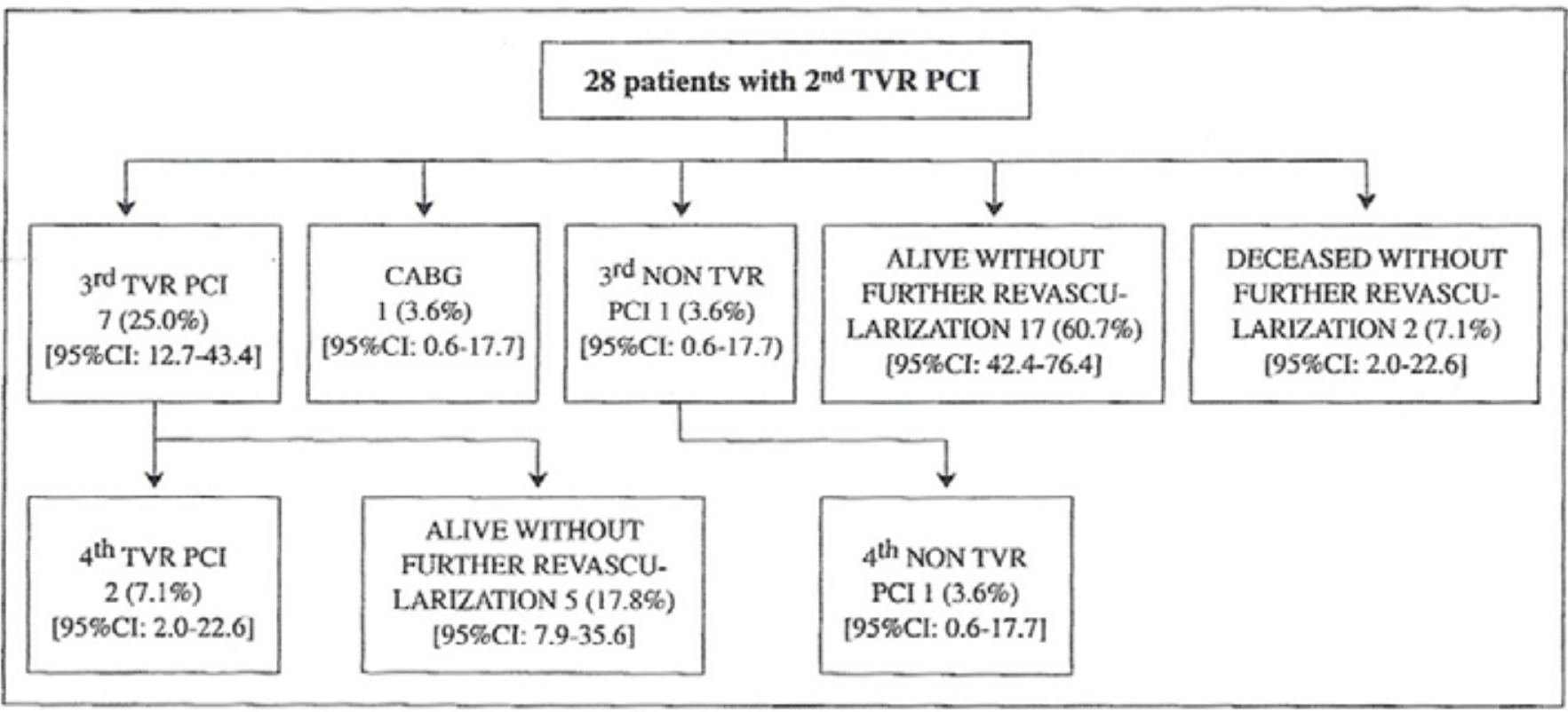

Figure 3.-Need of new revascularization procedures following a second repeat percutaneous coronary intervention, reported as N., \%, and 95\% confidence interval. CABG: coronary artery bypass grafting; NON TVR PCI: non target vessel revascularization percutaneous coronary intervention; TVR PCI: target vessel revascularization percutaneous coronary intervention.

low-up and maximal medical therapy remain pivotal in these patients, who can experience adverse events even several months or years following the index procedure and despite long periods of well being and freedom from angina or ischemia.

Diabetes mellitus is a recognised major risk factor for cardiovascular morbidity and mortality ${ }^{[12]}$ and an independent predictor of restenosis after coronary stenting. ${ }^{[10]}$ Several studies on the pathophysiology of diabetic coronary heart disease only partially explained the excess risk of adverse events by means of frequent comorbidities, conferring a crucial role to dysmetabolic features of diabetics condition itself and systemic inflammation. ${ }^{[2,11]}$

Unselected diabetics have a cardiovascular risk profile higher than patients enrolled in clinical trials. Their

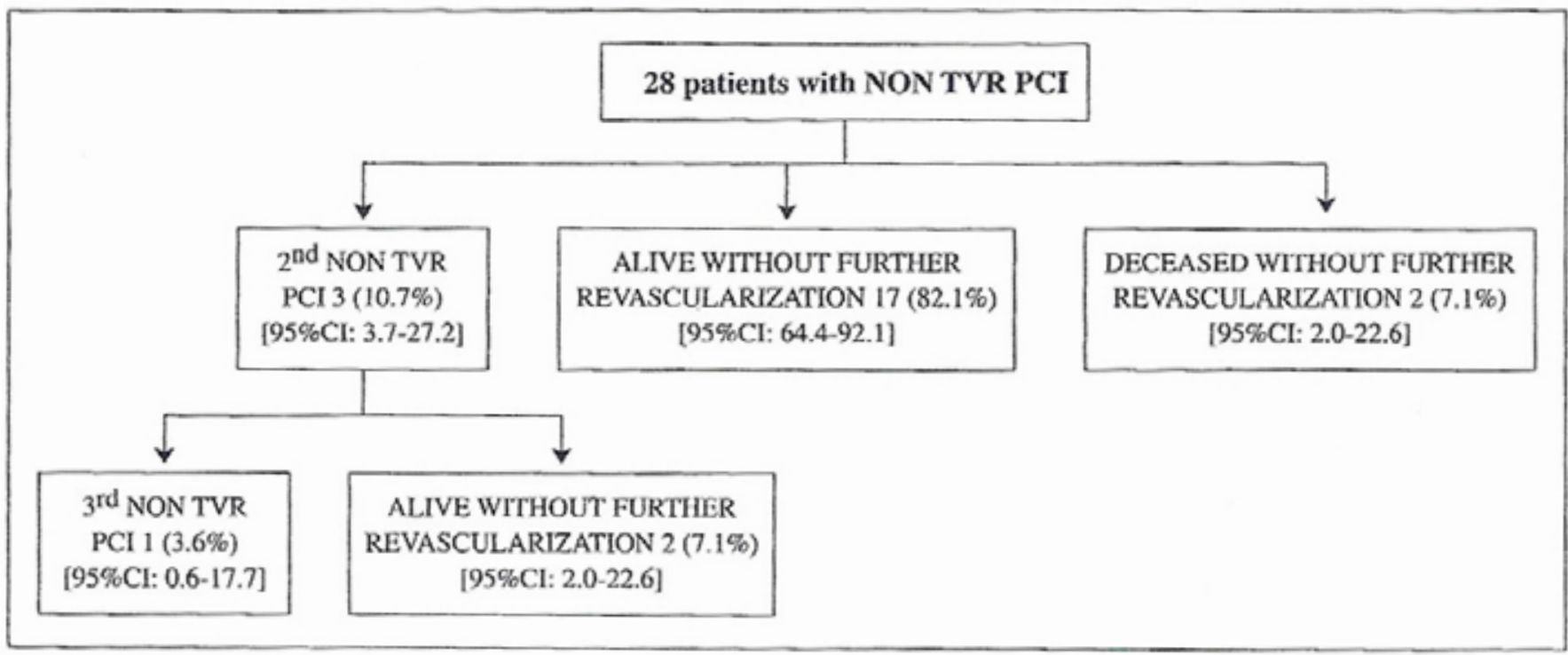

Figure 4.-Need of new revascularization procedures driven by coronary lesions located in epicardial vessels not treated during the index PCI, reported as N., \%, and $95 \%$ confidence interval. CABG: coronary artery bypass grafting; NON TVR PCI: non target vessel revascularization percutaneous coronary intervention; TVR PCI: target vessel revascularization percutaneous coronary intervention. 
clinical and angiographic complexity may be the plausible explanation for the relatively poor short and long term outcomes reported in this study.

Notwithstanding the rather complete revascularization obtained with the index procedure and secondary prevention medical care at discharge, more than one third of patients underwent a percutaneous or surgical re-intervention. Both in-stent restenosis and disease progression led to multiple subsequent revascularization, with no single sequence of interventions yielding complete freedom from adverse events.

Our study is unlike to randomized trials or observational studies comparing performance of drug-eluating and bare-metal stents. Instead, we examined safety and efficacy end points long after the occurrence of first adverse events. We thus obtained detailed information on the influence of diabetes on the response of these patients to percutaneous revascularization. Considerable recurrences of intervention for myocardial ischemia emerged at three-year follow-up, a long period to explore device properties, but brief when related to diabetes disease duration. The phenomenon evaluated in our analysis may thus assume greater proportions in the whole history of disease of diabetics.

Further studies based on the present design should investigate a more homogeneous population with a longer follow-up. Nonetheless, coronary revasculariza- tion maintain a prominent role in managing the heavy burden of coronary disease in diabetics, and the percutaneous approach is likely to extend its applications thanks to growing evidence for safety and efficacy of drug-eluting stents. This observational study, the first to examine the risk of recurrent averse events, adds new important information to our knowledge of coronary stenting in real-world diabetics. A randomized clinical trial clearly appears unsuited to explore an objective as complex as multiple repeat revascularizations.

Selection bias should not be a major internal study limitation because losses to follow-up were $7.9 \%$ an acceptable number given the all-comers population and the long term clinical follow-up. We cannot however exclude that primary outcome measures could be underestimated since only symptoms lead to repeat interventions and no systematic angiographic control nor testing for silent ischemia was planned.

\section{Conclusion}

In conclusion, this study, originally reporting on the risk of recurrent repeat revascularization events among diabetics treated with PCI, shows that repeat target vessel and non target vessel revascularization occurs frequently in this patient population, but can be managed in most cases in a safe and successful manner by means of repeat percutaneous intervention.

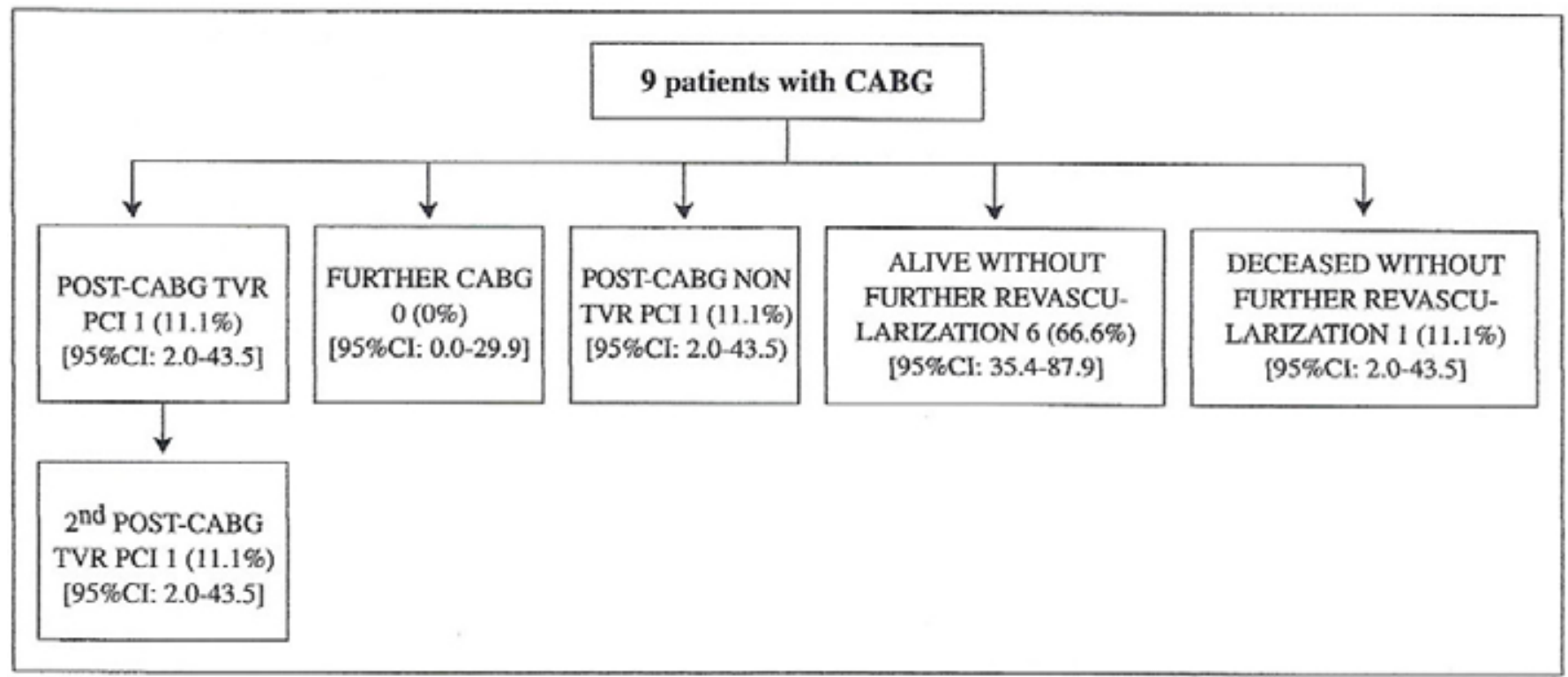

Figure 5.-Need of new revascularization procedures following a repeat surgical revascularization, reported as N., \%, and $95 \%$ confidence interval. CABG: coronary artery bypass grafting; NON TVR PCI: non target vessel revascularization percutaneous coronary intervention; TVR PCI: target vessel revascularization percutaneous coronary intervention. 


\section{References}

1. Stettler C, Allemann S., Juni P, Cull CA, Holmann RR, Egger M, et al. Glycemic control and macrovascular disease in types 1 and 2 diabetes mellitus : meta-analysis of randomized trials. Am Heart J 2006; 152:27-38.

2. Creager MA, Luscher TF, Cosentino F, Beckman JA, Diabetes and vascular disease : pathopfhysiology, clinical consequences, and medical thereply: Part I. Circulation 2003 ; 108: 1527-32.

3. Cubbon RM, Wheatcroft SB, Grant PJ, Gale CP, Barth JH, Sapford RJ et al. Evaluation of methods and management of acute coronary events investigators . temporal trends in mortality of patients with diabetes mellitus suffering acute myocardial infarction: a comparison of over 3000 patients between 1995 and 2003. Eur Heart J 2007; 28:540-5.

4. Flahearty JD, Davidson CJ, Diabetes and coronary revascularization . JAMA 2005; 293:1501-8.

5. Kirtane AJ, EllisSG, Dawkins KD, Colombo A, Grube E, Pompa JJ et al. Paclitaxel-eluting coronary stents in patients with diabetes mellitus. A Am Coll Cardiol 2008; 51:708-15.
6. American Diabetes Association,. Standards of medical care in diabetes -2008. Diabetes Care $2008 ; 31$.

7. ESC. Guidelins for percutaneous coronary intervention. The tsk force for the percutaneous coronary intervention of the European society of cardiology. European Heart Journal.2005; 26:804-47.

8. Thygesen K, Alpert JS, White HD, Joint ESC/ACCF/AHA/WHF Task force for the redefinition of myocardial infarction. Universal definition of myocardial infarction.Eur Heart J 2007; 28:2525-38.

9. Cutlip DE, Windecker S, Meheran R, Boam A, Cohen DJ, van es GA et al. Academic research consortium. Clinical and points in coronary stent trials: a case for standardized definition. Circulation 2007; 115:2344.51.

10. Cosgrave J, Melzi G, Biondi-ZoccaiGG, Airoldi F, Chieffo A, Sangiorgi GM et al. Drug-eluting stent restenosis : the pattern predicts the out come.J Am Coll Cardiol 2006; 47:2399-404.

11. Biondi-zoccai GG, Abbate A, Liuzzo G, Biasucci LM, Atherothrombosis, inflammation, and diabetes. J Am Coll Cardiol 2003;41:1071-7.

Received: $25 / 04 / 2013$

Accepted: 22/06/2013

Published: 15/07/2013

Disclosure and conflicts of interest:

Conflicts of interest were not reported.

\section{Corresponding author:}

Dr. Francesco Pollice

e-mail: francesco.pollice@tiscali.it 